Session 12-22

\title{
Indentation Analysis of Metal-Ceramic Multilayered Composites
}

\author{
Guanlin Tang, Yu-Lin Shen \\ Department of Mechanical Engineering \\ University of New Mexico
}

\begin{abstract}
Metal-ceramic nanolaminates are attractive materials because of their high strength and flexibility as well as their potential applications in functional devices. The effective elastic modulus of thin-film multilayers is normally measured by nanoindentation. In this paper, a numerical study was undertaken to study the elastic property of metal-ceramic multilayered composites derived from indentation testing. The model system features alternating thin films of aluminum (Al) and silicon carbide $(\mathrm{SiC})$, free from any effect due to the underlying substrate. The true effective elastic response of the laminate was represented by a homogenized anisotropic material, with its modulus values obtained by simulating overall loading of the structure. A two-dimensional axisymmetric model with a rigid conical indenter was created using Abaqus 6.5. Two finite element modeling simulations of instrumented indentation, one with real multilayered features and the other with a homogenized anisotropic material, were then employed to calculate the indentation-derived modulus using the unloading portion of the load-displacement curve. The numerical results from indenting the homogenized composite (with the built-in multilayer property) and from indenting the real multilayers (with $\mathrm{Al}$ and $\mathrm{SiC}$ layers explicitly accounted for) were compared. It was found that, with a large number of alternating metal-ceramic layers included in the model, an indentation depth equal to several initial layer thicknesses is sufficient to yield a representative elastic response. The indentation-derived effective modulus was found to be close to the out-of-plane overall modulus of the multilayer composite. A limited set of analyses on the effect of the substrate material has also been conducted.
\end{abstract}

\section{Introduction}

Composites consisting of alternating metal and ceramic layers at the nano- and micro-scales are attractive materials because of their high strength, high toughness, damage tolerance, as well as their potential applications in functional devices ${ }^{1-14}$. Traditional mechanical testing methods for bulk materials are not easily used on small-scale structures. Characterization of the effective mechanical properties of the laminates has been conducted by instrumented indentation. When the indentation depth is sufficiently large, the measured elastic modulus and hardness values may be representative of the structure as a whole. However, the relationship between the indentation-derived mechanical properties and the "true" composite properties is still not well understood". A continuum-based numerical study focusing on the effective hardness of multilayers has been reported ${ }^{16}$. Under the ideal elastic-perfectly plastic assumption, it was shown that the indentation hardness, upon conversion to plastic flow stress, underestimates the overall strength of the composite. This work demonstrated the potential uncertainty in assessing intrinsic material properties utilizing the indentation test. 
In the present study, we have focused on the effective elastic modulus of the metal-ceramic multilayers obtained from indentation. Our model system consists of alternating layers of aluminum (Al) and silicon carbide ( $\mathrm{SiC}$ ). The true effective elastic response of the composite was represented by a homogeneous anisotropic material. The elastic constants of the composite were obtained by a combination of analytical and numerical means under uniaxial compression loading. Finite element modeling of indentation into the homogeneous material was then employed to calculate the indentation-derived modulus (using the unloading portion of the load-displacement curve). The indentation-derived modulus and the "true" composite modulus were compared. In addition, finite element modeling of indentation was conducted with the multilayers modeled explicitly. The primary objectives of this work are to determine if the true elastic response of the multilayers can be extracted from instrumented indentation, and to examine how the indentation-derived modulus compares with the actual anisotropic elastic properties of the composite.

\section{Numerical Model}

\section{Overall Elastic Properties}

A schematic of the multilayer structure, composed of a large number of alternating metal and ceramic layers, is shown in Fig. 1. The individual layers are isotropic, each with an in-plane dimension (along the 1- and 3-directions) much greater than the out-of-plane dimension (the 2direction). All interfaces between adjacent layers are assumed to be perfectly bonded so the displacement field across the interface is continuous. Although the individual layer thickness may be conceived to be in the micrometer or nanometer range, there is no intrinsic length scale involved in the present scheme.

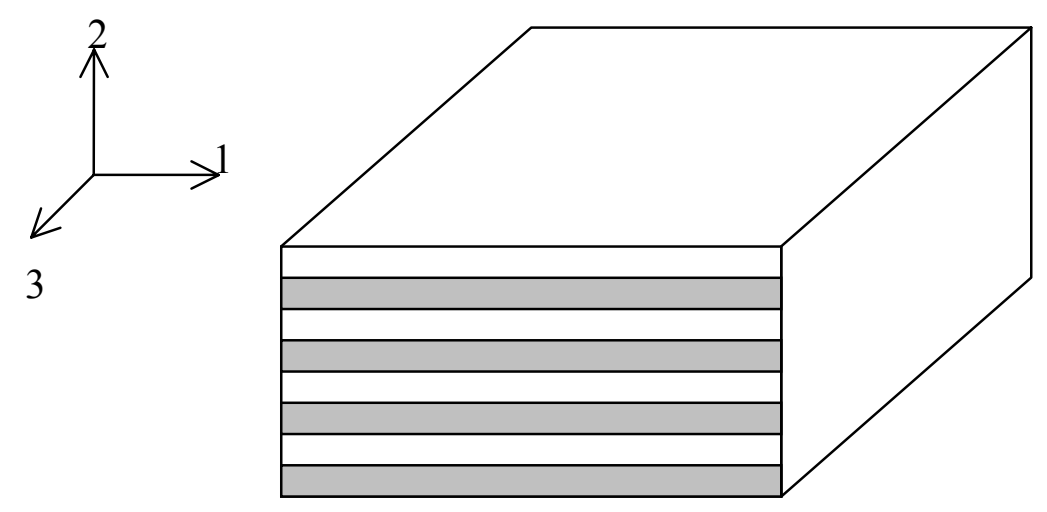

Figure 1. Schematic showing the alternating metal-ceramic layers considered in this study

If the composite in Figure 1 is treated as a homogeneous material, its anisotropic elastic properties can be represented by a set of elastic constants. It is convenient to start with the generalized Hooke's law for an orthotropic material system: 


$$
\left[\begin{array}{c}
\varepsilon_{11} \\
\varepsilon_{22} \\
\varepsilon_{33} \\
\gamma_{12} \\
\gamma_{13} \\
\gamma_{23}
\end{array}\right]=\left[\begin{array}{cccccc}
1 / E_{11} & -v_{21} / E_{22} & -v_{31} / E_{33} & 0 & 0 & 0 \\
-v_{12} / E_{11} & 1 / E_{22} & -v_{32} / E_{33} & 0 & 0 & 0 \\
-v_{13} / E_{11} & -v_{23} / E_{22} & 1 / E_{33} & 0 & 0 & 0 \\
0 & 0 & 0 & 1 / G_{12} & 0 & 0 \\
0 & 0 & 0 & 0 & 1 / G_{13} & 0 \\
0 & 0 & 0 & 0 & 0 & 1 / G_{23}
\end{array}\right]\left[\begin{array}{c}
\sigma_{11} \\
\sigma_{22} \\
\sigma_{33} \\
\sigma_{12} \\
\sigma_{13} \\
\sigma_{23}
\end{array}\right],
$$

where $\varepsilon, \gamma, \sigma, E, G$ and $v$ represent the normal strain, shear strain, stress, Young's modulus, shear modulus and Poisson's ratio, respectively. The coordinate axes are based on those defined in Figure 1. For an orthotropic material the following relations hold true ${ }^{17}$ :

$\frac{v_{12}}{E_{11}}=\frac{v_{21}}{E_{22}}, \frac{v_{13}}{E_{11}}=\frac{v_{31}}{E_{33}}, \frac{v_{23}}{E_{22}}=\frac{v_{32}}{E_{33}}$.

Therefore there are a total of 9 independent elastic constants (which may be taken as $E_{11}, E_{22}, E_{33}$, $v_{12}, v_{13}, v_{23}, G_{12}, G_{13}$ and $G_{23}$ ). The multilayered structure considered here is a special case of the orthotropic material: It is transversely isotropic along the 13-plane. As a consequence,

$E_{11}=E_{33}, G_{12}=G_{23}, v_{21}=v_{23}, v_{12}=v_{32}, v_{13}=v_{31}, G_{13}=\frac{E_{11}}{2\left(1+v_{13}\right)}$.

There are now only 5 independent elastic constants, which may be chosen as $E_{11}, E_{22}, v_{12}, v_{13}$ and $G_{12}$. These 5 constants are determined following the approach outlined below.

A simple way to compute the magnitudes of $E_{11}$ and $E_{22}$ of the composite, is to use the relations based on the isostrain condition (Voigt model) and isostress condition (Reuss model), respectively. The composite modulus $E_{c}$ in the isostrain condition $\left(E_{11}\right)$ is:

$E_{c}=E_{A l} f_{A /}+E_{S i C} f_{S i C}$,

while in the isostress case $\left(E_{22}\right)$,

$$
E_{c}=\frac{1}{\frac{f_{A l}}{E_{A l}}+\frac{f_{S i C}}{E_{S i C}}} \text {. }
$$

Here $f$ represents the volume fraction of the constituents denoted by the subscripts. It should be noted that Eqs. (4) and (5) are derived under a one-dimensional assumption so care must be taken in directly applying them to the material considered here. For instance, when the layered composite is subject to loading along the 1-direction, uneven deformation in the 3-direction between layers will occur due to the different Poisson's ratios of $\mathrm{Al}$ and $\mathrm{SiC}$. This will generate stresses in the 3direction and, in turn, will affect the stresses in the 1-direction and thus the longitudinal composite modulus, $E_{11}$. Similarly, when the loading is along the 2-direction, the strains along the same direction in the two materials will not be the same. Unequal lateral deformations between the layers will occur, which will in turn affect stresses in the 2-direction, and thus, the transverse composite modulus, $E_{22}$. Thus, depending on the magnitude of the elastic constants, significant errors may exist if one uses these one-dimensional approximations (Eqs. (4) and (5)). As a consequence, in the present study we use finite element modeling of overall uniaxial loading of the multilayers to accurately determine the necessary elastic constants, as described below. 


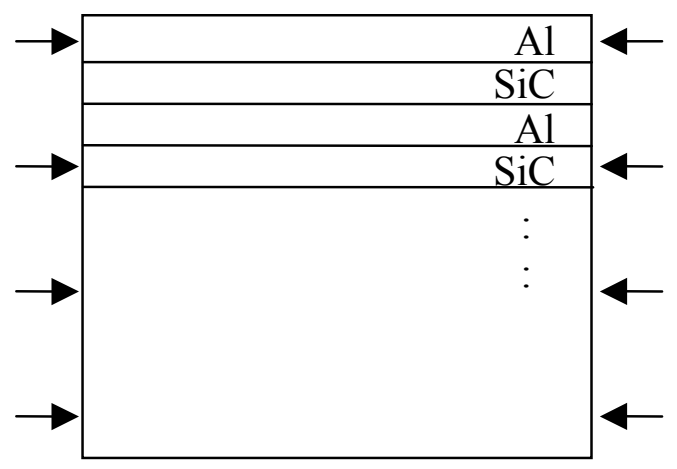

(a)

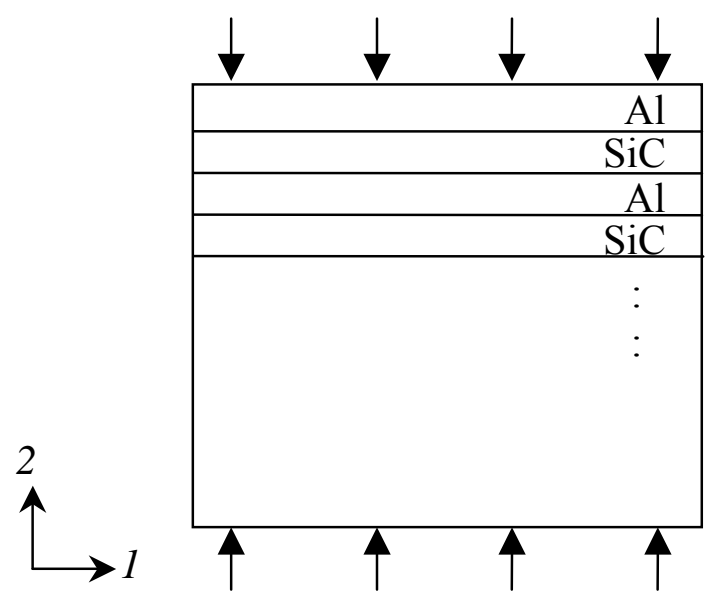

(b)

Figure 2. Schematics showing the (a) longitudinal and (b) transverse loading configurations for modeling the overall elastic response of the multilayered composite

Figure 2 shows a schematic of compressive loading of the multilayered structure. The composite Young's moduli were calculated from the ratio of stress and strain along the direction of interest, and the composite Poisson's ratios were calculated from the respective strain ratios. In the actual numerical model only two representative layers were included with appropriate boundary conditions imposed such that a periodic stacking along the transverse (out-of-plane) direction and an infinite dimension in the longitudinal (in-plane) direction were ensured. This was accomplished by setting the top and bottom boundaries to remain horizontal and the side boundaries to remain vertical during deformation, similar to the unit-cell approach for simulating particle-matrix composite systems ${ }^{18,19}$. The calculated composite response is the "true" effective property of the entire multilayer structure with all three-dimensional features accounted for. The longitudinal loading configuration in Figure 2(a) was used for obtaining $E_{11}, v_{12}$ and $v_{13}$, and the transverse loading configuration in Figure 2(b) was used for obtaining $E_{22}$. 
The remaining elastic constant to be determined is $G_{12}$. It can be directly obtained from the analytical expression

$G_{12}=\left[\frac{f_{A l}}{G_{A l}}+\frac{f_{S i C}}{G_{S i C}}\right]^{-1}$

which is based on the equivalent shear stress condition ${ }^{20}$. The five independent elastic constants are then used as input parameters for simulating indentation of the "homogenized" $\mathrm{Al} / \mathrm{SiC}$ composite.

\section{Indentation Modeling}

Indentation modeling was based on an axisymmetric model featuring a rigid conical indenter. Two models of the same geometry were considered: one with the $\mathrm{Al}$ and $\mathrm{SiC}$ layers explicitly accounted for and the other a homogenous block possessing the anisotropic elastic property of the $\mathrm{Al} / \mathrm{SiC}$ composite. They are henceforth referred to as the "multilayer model" and "homogenized model," respectively. Note that the two models have exactly the same overall elastic behavior. Figure 3 shows the schematic of the model of indentation into the multilayer. The left boundary is treated as the symmetry axis. The semi-angle of the conical indenter is $70.3^{\circ}$, resulting in a same projected area as that of a Berkovich indenter ${ }^{21}$. In the upper portion of the model there are a total of 100 alternating layers of $\mathrm{Al}$ and $\mathrm{SiC}$. Although this model is intended to represent the real composite with an "infinite" number of layers, a homogenized $\mathrm{Al} / \mathrm{SiC}$ material having the anisotropic composite property is placed underneath the 100 explicit layers. The main purpose of this is to improve computational efficiency by allowing a coarser mesh size in the less deformed lower region. Very small elements have to be employed within the layered region to sufficiently resolve the geometry-limited deformation field.

Although there is no intrinsic length scale in the analysis, it is convenient to associate the model with specific physical dimensions. The overall size of the entire specimen is taken as $40 \mu \mathrm{m}$ in lateral span (radius) and $43 \mu \mathrm{m}$ in height. The region containing 100 layers of $\mathrm{Al}$ and $\mathrm{SiC}$ has a total thickness of $5 \mu \mathrm{m}$. In the case of the homogenized model, both the Al and SiC layers in Figure 3 were simply replaced by the homogenized $\mathrm{Al} / \mathrm{SiC}$ composite. In the simulation the maximum indentation depth considered is within $2 \%$ of the total height and radius of the specimen, so the edge effect due to the side and bottom boundaries was negligible (according to our preliminary calculations). During deformation the left boundary is allowed to move only in the 2-direction. The bottom boundary is allowed to move only in the 1-direction. The right boundary is not constrained. The top boundary is also free to move, except when contact with the indenter is established, the surface portion engaged by the indenter is restricted to follow the indenter contour. The coefficient of friction at the contact is taken to be 0.1 , which is a typical value for the diamond/metal contact pair $^{22,23}$. 


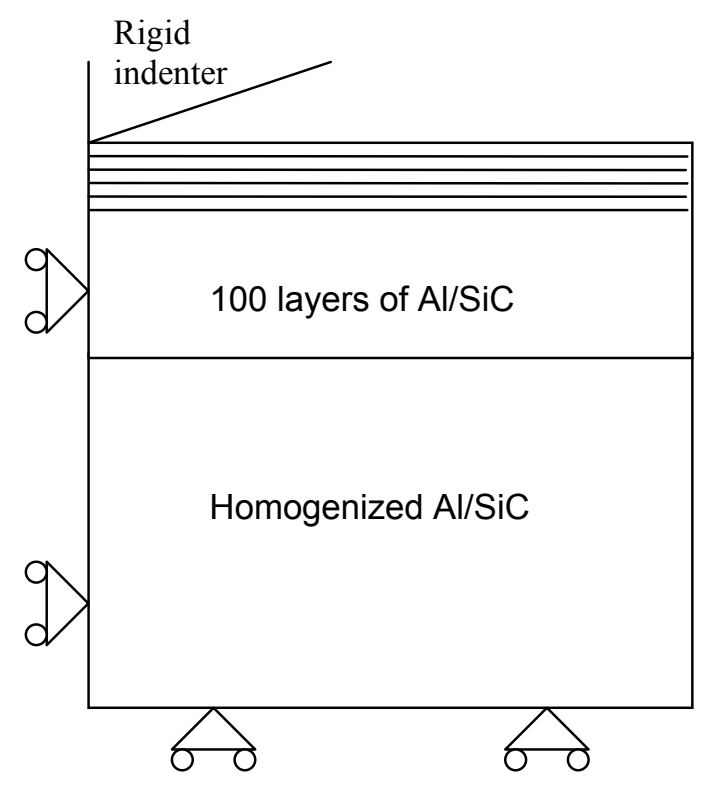

Figure 3. Schematic showing the multilayer model and the boundary conditions for indentation modeling. The specimen and indenter both possess axial symmetry about the left boundary. The rigid indenter has a semi-angle of $70.3^{\circ}$

The indentation-derived elastic modulus from the simulation is obtained according to the method proposed by Oliver and Pharr ${ }^{24}$. The method is based on the expression:

$S=\beta \frac{2}{\sqrt{\pi}} E_{r} \sqrt{A}$

where $S$ is contact stiffness obtained from the initial unloading slope of an indentation loaddisplacement curve, $A$ is the projected contact area at the onset of unloading, $\beta$ is an indenter geometry-dependent dimensionless parameter close to unity, and $E_{r}$ is the reduced modulus given by $\frac{1}{E_{r}}=\frac{1-v^{2}}{E}+\frac{1-v_{i}^{2}}{E_{i}}$.

In Eq. (8) $E$ and $v$ are the Young's modulus and Poisson's ratio of the material being tested, and $E_{i}$ and $v_{i}$ are the Young's modulus and Poisson's ratio of the indenter. In our simulation the parameter $\beta$ was first calibrated with a pure Al body of the same geometry, and a value of 1.06 was determined which was followed in all subsequent analyses. When calculating the projected contact area $A$, the last nodal point on the top surface in contact with the indenter was identified in the deformed finite element mesh so the effect of pileup resulting from the indentation was taken into account. The calculation of indentation-derived modulus $E$ from $E_{r}$ requires a known Poisson's ratio of the composite. Here we used the $v_{12}$ value obtained from the modeling of overall uniaxial loading of the $\mathrm{Al} / \mathrm{SiC}$ composite, as described above.

The finite element program ABAQUS (Version 6.5, Dassault Systemes Simulia Corp., Providence, RI) was employed in all calculations. A total of 174,266 linear elements were used in the model, with a finer mesh size near the upper-left corner. The input Young's moduli for Al and SiC were those measured from experiments of the single-layer film ${ }^{6}$. The individual materials were treated as 
isotropic elastic-perfectly plastic, with the input plastic response of both materials based on the tensile loading data of single-layer $\mathrm{Al}^{6}$. In a separate preliminary numerical study, we have confirmed that, under the current model configuration, the indentation-derived elastic modulus is essentially independent of the input plastic behavior in the finite element model. This is also consistent with other theoretical and/or numerical studies ${ }^{25,26}$. Therefore the present approach is deemed appropriate, and was able to avoid the potential numerical problem when attempting to indent extremely hard layers to a sufficient depth. The Young's modulus $E$ and Poisson's ratio $v$, used as input parameters in all the finite element modeling, were: $E_{\mathrm{Al}}=59 \mathrm{GPa}, E_{\mathrm{SiC}}=277 \mathrm{GPa}, v_{\mathrm{Al}}$ $=0.33, v_{\mathrm{SiC}}=0.17$. In this work the calculation of the overall anisotropic elastic property for the homogenized model incorporates the entire span of $\mathrm{Al} / \mathrm{SiC}$ volume (thickness) ratio from zero to one. Three specific $\mathrm{Al} / \mathrm{SiC}$ volume ratios were selected for the indentation modeling: A150/SiC50, $\mathrm{A} 125 / \mathrm{SiC} 75$ and $\mathrm{A} 175 / \mathrm{SiC} 25$ (here the numbers represent the thickness of the individual layer, in $\mathrm{nm})$.

In addition to the above described "infinite layers" model, a different model system which has 41 alternating layers of $\mathrm{Al}$ and $\mathrm{SiC}$ on a silicon ( $\mathrm{Si}$ ) substrate was also considered. The overall model geometry and the boundary conditions for the indentation simulation were the same as the "infinite layers" model. Instead of a rigid indenter, an elastic diamond indenter is used. Figure 4 shows the schematic of the model. The Young's modulus $E$ and Poisson's ratio $v$, used as input parameters for the Si substrate and diamond indenter, were: $E_{\mathrm{Si}}=187 \mathrm{GPa}, v_{\mathrm{Si}}=0.28$, $v_{\text {Diamond }}=1141 \mathrm{GPa}, v_{\text {Diamond }}$ $=0.07$. We focus on how the substrate would affect the indentation derived elastic modulus of the $\mathrm{Al} / \mathrm{SiC}$ multilayers.

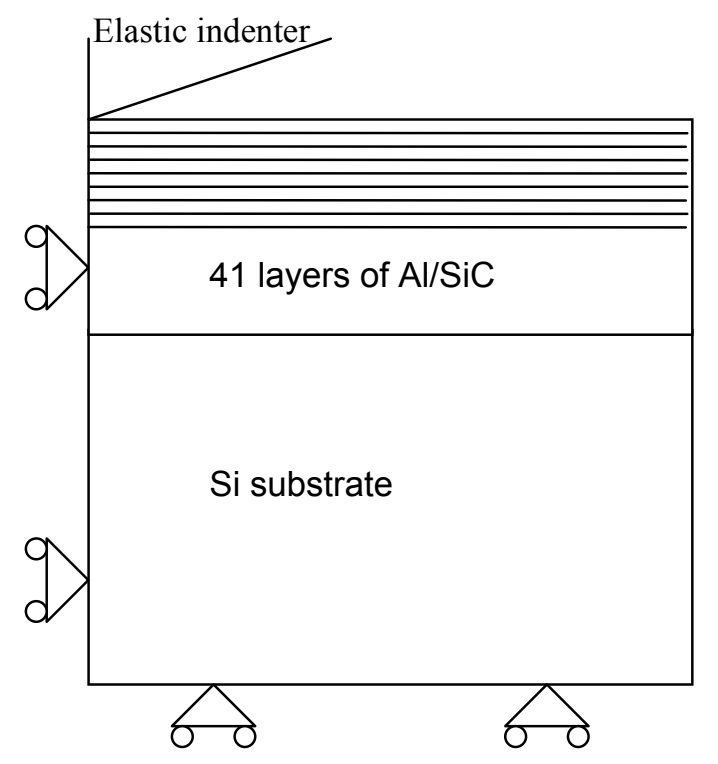

Figure 4. Schematic showing the multilayers on a Si substrate and the boundary conditions for indentation modeling. The specimen and indenter both possess axial symmetry about the left boundary. The rigid indenter has a semi-angle of $70.3^{\circ}$. 


\section{Results and Discussion}

\section{Overall Elastic Properties}

We first present the overall elastic properties of the $\mathrm{Al} / \mathrm{SiC}$ multilayer composite, on the basis of the approach described in Section 2.1. Figure 5 shows the numerically modeled Young's modulus values as a function of volume fraction of $\mathrm{SiC}$. For comparison purposes the modulus values given by the one-dimensional approximation (Eqs. (4) and (5)) are also included in the figure. It can be seen that the difference between the longitudinal modulus $\left(E_{11}\right)$ and transverse modulus $\left(E_{22}\right)$ of the $\mathrm{Al} / \mathrm{SiC}$ multilayers is quite large. In general the numerically modeled $E_{11}$ values are close to those given by Eq. (4), but there is a significant discrepancy between the numerical $E_{22}$ values and those from Eq. (5). The inaccuracy of applying the one-dimensional approximation to composite modulus of the metal/ceramic multilayers is thus illustrated.

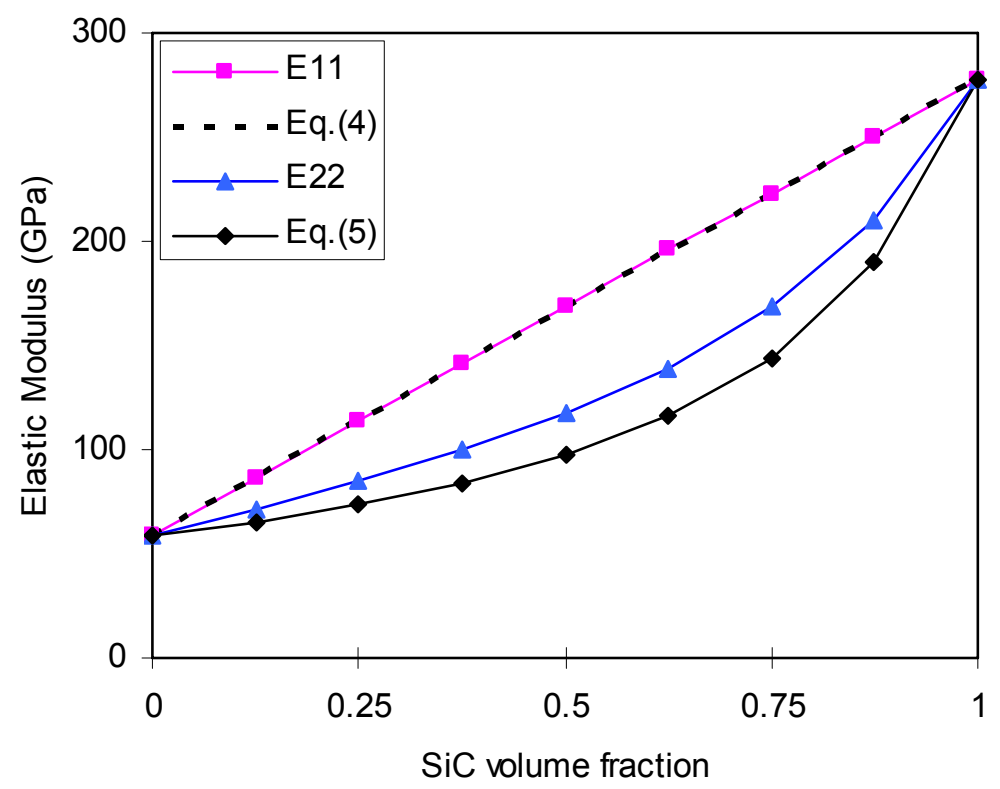

Figure 5. Numerical result showing the overall Young's modulus along the longitudinal direction $\left(E_{11}\right)$ and transverse direction $\left(E_{22}\right)$ of the $\mathrm{Al} / \mathrm{SiC}$ multilayers. For comparison the modulus values based on the one-dimensional assumption (Eqs. (4) and (5)) are also included.

The overall elastic response is needed as input properties for the homogenized composite during indentation modeling, for both the entire test material in the "homogenized model" or for the lower portion of the "multilayer model." The five independent elastic constants for each of the three volume fractions used in the indentation modeling are listed in Table 1. 
Table 1. Five independent elastic constants for the three composite models: A175/SiC25, A150/SiC50 and A125/SiC75

\begin{tabular}{lccccc}
\hline Composite & $E_{11}(\mathrm{GPa})$ & $E_{22}(\mathrm{GPa})$ & $v_{12}$ & $v_{13}$ & $G_{12}(\mathrm{GPa})$ \\
\hline $\mathrm{A} 175 / \mathrm{SiC} 25$ & 114.2 & 84.8 & 0.3215 & 0.2357 & 27.8 \\
$\mathrm{~A} 150 / \mathrm{SiC} 50$ & 168.7 & 116.9 & 0.2789 & 0.2002 & 37.4 \\
$\mathrm{~A} 125 / \mathrm{SiC} 75$ & 222.9 & 168.3 & 0.2265 & 0.1815 & 56.8 \\
\hline
\end{tabular}

\section{Indentation Analysis of Al50/SiC50 Composite}

We now present the results from indentation modeling. Figure 6 shows a representative indentation load-displacement curve for the multilayer model of A150/SiC50, with a maximum indentation depth at $100 \mathrm{~nm}$ (equal to two initial layer thicknesses in this case). All other models show the same qualitative features. The initial slope during unloading was used to calculate the indentation-derived elastic modulus of the composite.

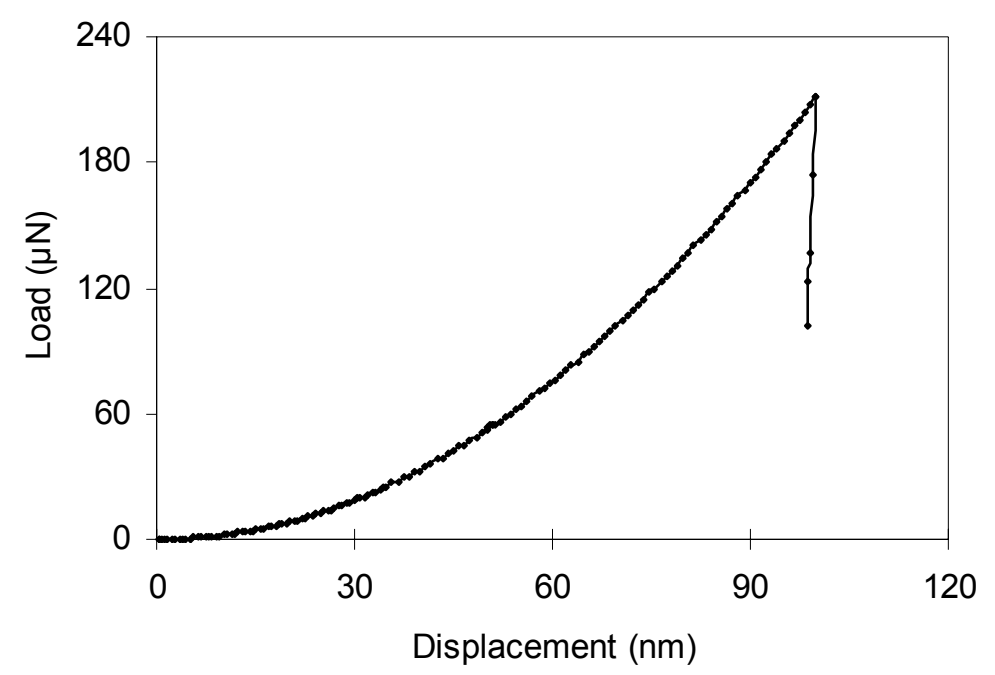

Figure 6. A representative indentation load-displacement response obtained from the finite element modeling. This curve corresponds to the multilayer model $\mathrm{Al50} / \mathrm{SiC} 50$, with the maximum indentation depth at $100 \mathrm{~nm}$.

Figure 7 shows the numerical results of the indentation-derived elastic modulus as a function of indentation depth, for the case of A150/SiC50. The maximum indentation depth shown is $700 \mathrm{~nm}$, which corresponds to a depth of 14 initial layer thicknesses. For reference the modeled result for a pure Al specimen is also included, which shows a constant modulus value over the range of indentation depth considered. The $\mathrm{A} 150 / \mathrm{SiC} 50$ composite response is represented by three curves in Figure 7: one of the homogenized model and two of the multilayer model. The difference in the two multilayer cases is the material used as the topmost layer, $\mathrm{Al}$ or $\mathrm{SiC}$. 


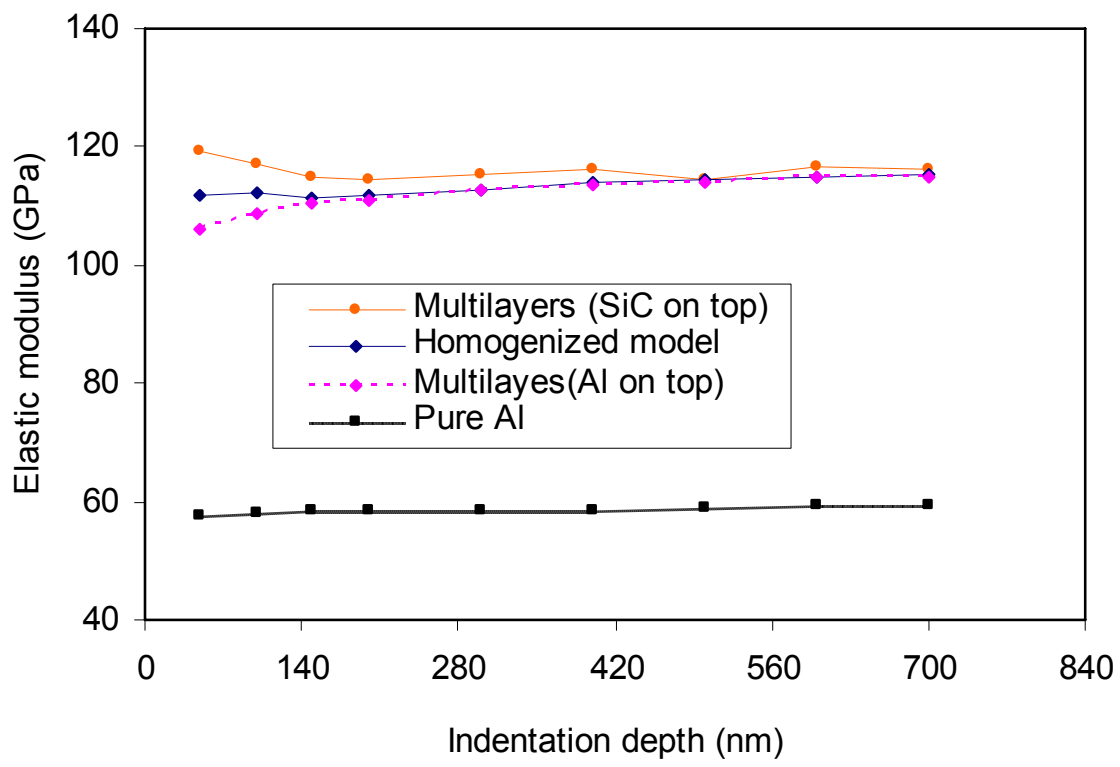

Figure 7. Indentation-derived elastic modulus, obtained from the finite element analysis, of the $\mathrm{Al} 50 / \mathrm{SiC} 50$ composite as a function of the indentation depth. The result of pure $\mathrm{Al}$ is also included for reference. In addition to the homogenized model, two curves for the multilayer model are presented: one with $\mathrm{Al}$ being the topmost layer and the other with $\mathrm{SiC}$

being the topmost layer.

It can be seen from Figure 7 that, when the indentation is relatively shallow, the response of the multilayer model is sensitive to the first-layer material in contact with the indenter. If Al is the topmost layer, the elastic modulus is relatively low and vice versa. The three curves tend to converge when the indentation depth is large (beyond about $400-500 \mathrm{~nm}$, or 8-10 initial layer thicknesses). The merging of the curve of the multilayer model (with Al on top) to that of the homogenized model appears at an even smaller indentation depth. We can conclude that, when performing nanoindentation tests on metal-ceramic multilayers consisting of a large number of layers, a depth of indentation beyond several initial layer thicknesses may be sufficient for generating the "true" elastic response of the composite.

\section{Other Al/SiC Thickness Ratios}

Next we consider results of the A175/SiC25 and A125/SiC75 composites. Only the multilayer model with Al being the topmost layer is presented here. Figure 8 shows the modeled indentation-derived elastic modulus as a function of indentation depth. The result of A150/SiC50 is also included in the figure for comparison. The modulus variation follows the same trend in all three cases. With a sufficient indentation depth a steady modulus value can be reached. The numerical values are 83.9 $\mathrm{GPa}, 114.9 \mathrm{GPa}$, and 167.9 $\mathrm{GPa}$ for $\mathrm{Al75} / \mathrm{SiC} 25, \mathrm{~A} 150 / \mathrm{SiC} 50$, and $\mathrm{A} 125 / \mathrm{SiC} 75$, respectively. 


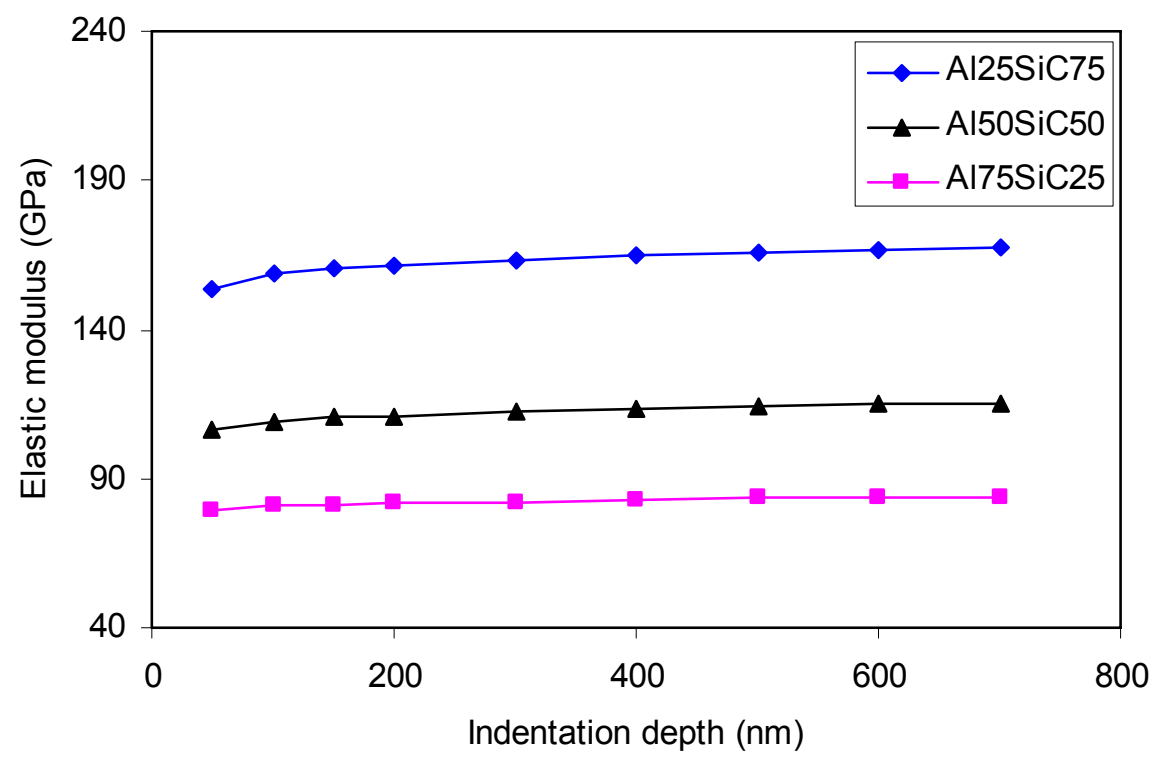

Figure 8. Indentation-derived elastic modulus as a function of the indentation depth for the composites $\mathrm{Al} 75 / \mathrm{SiC} 25$, $\mathrm{A} 150 / \mathrm{SiC} 50$ and $\mathrm{A} 125 / \mathrm{SiC} 75$

The correlation between the indentation-derived elastic modulus, which is a single value, and the effective anisotropic elastic property of the composite, is shown in Figure 9. Here the modulus values (at the deepest indentation considered) for the three multilayer composites A175/SiC25, $\mathrm{A} 150 / \mathrm{SiC} 50$ and $\mathrm{A} 125 / \mathrm{SiC} 75$ are overlaid on the plot showing the variation of composite $E_{11}$ and $E_{22}$ with the $\mathrm{SiC}$ volume fraction. It is evident that the three discrete points in Figure 9 are very close to the $E_{22}$ curve. This observation suggests that the modulus obtained from indentation may be a good representation of the overall transverse elastic modulus of the multilayers. 


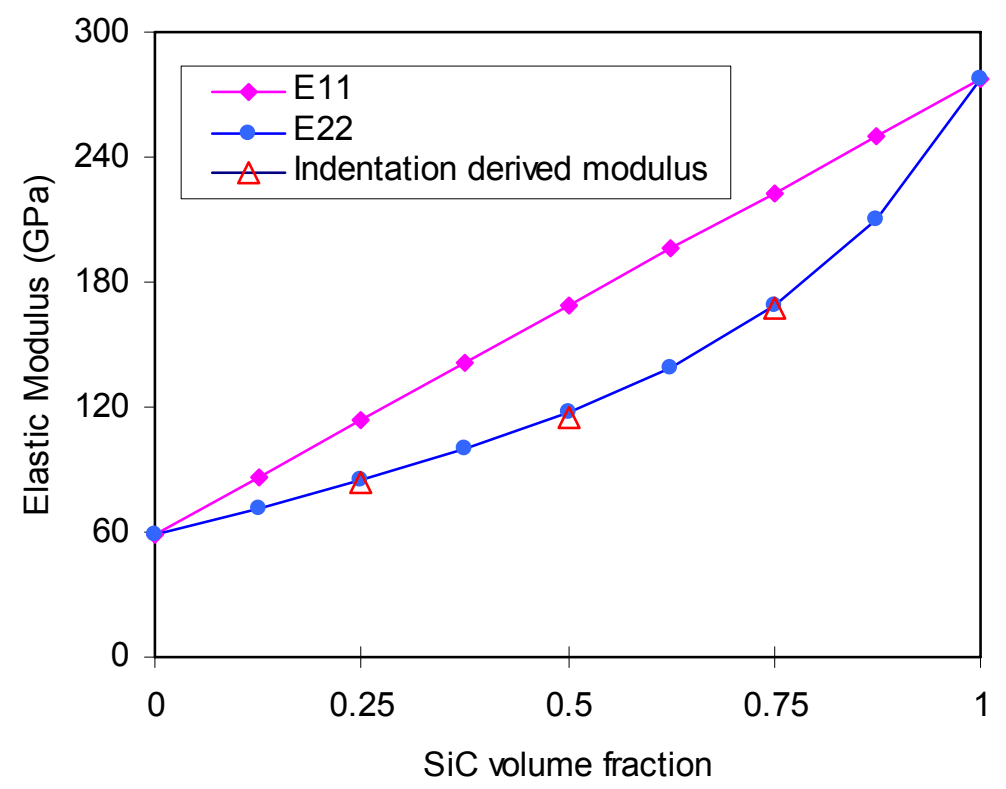

Figure 9. Comparison of the overall longitudinal modulus $E_{11}$ and transverse modulus $E_{22}$ with the indentation-derived modulus values of the composites $\mathrm{A} 175 / \mathrm{SiC} 25, \mathrm{Al} 0 / \mathrm{SiC} 50$ and $\mathrm{A} 25 / \mathrm{SiC} 75$

\section{Further Discussion}

The present study aims at gaining a fundamental understanding of the elastic property of metalceramic laminates measured from the instrumented indentation technique, through a systematic continuum-based numerical analysis. The composite geometry of concern consists of essentially "infinite" number of alternating metal and ceramic layers, so the possible effect of the underlying substrate material was avoided. In reality this condition is not always met. Here we consider the model containing 41 layers of $\mathrm{Al} / \mathrm{SiC}$ on a $\mathrm{Si}$ substrate. Figure 10 shows the numerical results of the indentation-derived elastic modulus as a function of indentation depth. The maximum indentation depth is 700nm (14 layers). It does not show a constant modulus value over the range of indentation depth considered. Rather, the modulus value continues to increase as the indentation goes deeper, suggesting the significance of the substrate stiffness effect. It appears to be very difficult to prevent the substrate material from contributing to the measured effective modulus of the multilayers. A thorough understanding of the interaction between the substrate and the layers, as well as their combined effect on indentation response, will require further investigations. 


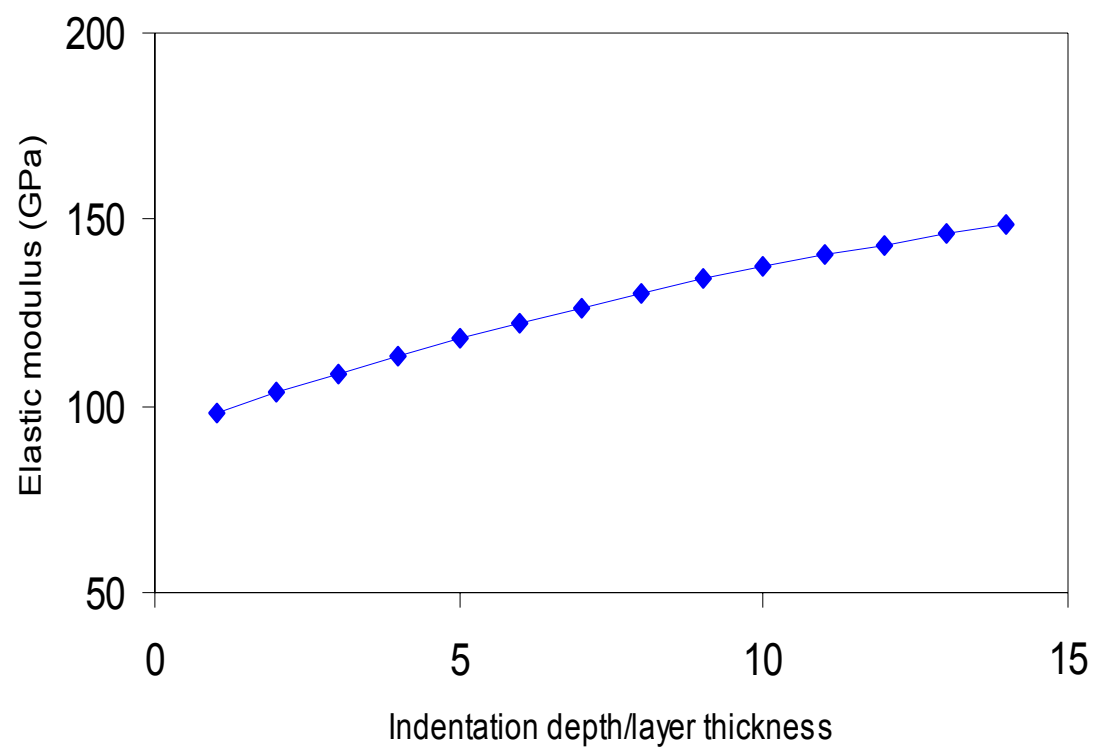

Figure 10. Elastic modulus from indentation modeling as a function of the indentation depth for the composites of 41 layer $\mathrm{A} 150 / \mathrm{SiC} 50$ on $\mathrm{Si}$

\section{Conclusions}

A systematic finite element analysis was carried out to study the effective elastic modulus of metalceramic multilayers obtained from the indentation technique. The composite structure considered consists of a large number of layers and is free from any substrate effect. Using the $\mathrm{Al} / \mathrm{SiC}$ multilayers as a model system, the modulus values, calculated from the modeled indentation loaddisplacement curve during unloading, were compared with the overall elastic property of the composites. It was found that, when the indentation reaches beyond about 8-10 initial thicknesses of the individual layers, the indentation response becomes representative of the entire composite. The multilayer modulus derived from the indentation test is consistent with the overall transverse (outof-plane) modulus of the composite. The findings apply to a wide range of relative thicknesses between the metal and ceramic layers. When a fewer number of $\mathrm{Al} / \mathrm{SiC}$ layers exists in the model above a Si substrate, however, the indentation-derived modulus was found to increase monotonically with the indentation depth. The influence of the substrate material is significant throughout the range of indentation depth considered.

\section{References}

1. Alpas, A. T., Embury, J. D., Hardwick, D. A. and Springer, R. W.: The mechanical properties of laminated microscale composites of A1/Al2O3. J. Mater. Sci. 25, 1603-1609 (1990).

2. Arcos, T. de los, Oelhafen, P., Aebi, U., Hefti, A., Duggelin, M., Mathys, D., Guggenheim, R.: Preparation and characterization of TiN-Ag nanocomposite films. Vacuum. 67, 463-470 (2002).

3. Chou, T. C., Nieh, T. G., Tsui, T. Y., Pharr, G. M., Oliver, W. C.: Mechanical properties and microstructures of metal ceramic microlaminates. 1. Nb/MoSi2 systems. J. Mater. Res. 7, 2765-2773 (1992).

4. Chou, T. C., Nieh, T. G., McAdams, S. D., Pharr, G. M., Oliver, W. C.: Mechanical properties and microstructures 
of metal ceramic microlaminates. 2. A Mo/Al2O3 system. J. Mater. Res. 7, 2774-2784 (1992).

5. Daia, M. Ben, Aubert, P., Labdi, S., Sant, C., Sadi, F. A., Houdy, P., Bozet, J. L.: Nanoindentation investigation of Ti/TiN multilayers films. J. Appl. Phys. 87, 7753-7757 (2000).

6. Deng, X., Chawla, N., Chawla, K. K., Koopman, M., Chu, J. P.: Mechanical behavior of multilayered nanoscale meta-ceramic composites. Adv. Eng. Mater. 7, 1099-1108 (2005).

7. Lee, J. H., Kim, W. M., Lee, T. S., Chung, M. K., Cheong, B. K., Kim, S. G.: Mechanical and adhesion properties of Al/AlN multilayered thin films. Surface Coatings Technol. 133, 220-226 (2000).

8. Liu, C. H., Li, W.-Z., Li, H.-D.: TiC/metal nacrous structures and their fracture toughness increase. J. Mater. Res. 11, 2231-2235 (1996).

9. Lousa, A., Romero, J., Martinez, E., Esteve, J., Montala, F., Carreras, L.: Multilayered chromium/chromium nitride coatings for use in pressure die-casting. Surface Coatings Technol. 146, 268-273 (2001).

10. Mearini, G. T.,Hoffman, R. W.: Tensile properties of aluminum alumina multilayered thin films. J. Electronic Mater. 22, 623-629 (1993).

11. Northwood, D. O., Alpas, A. T.: Mechanical and tribological properties of nanocrystalline and nanolaminated surface coatings. Nanostructured Mater. 10, 777-793 (1998).

12. Phillips, M. A., Clemens, B. M., Nix, W. D.: Microstructure and nanoindentation hardness of Al/Al3Sc multilayers. Acta Mater. 51, 3171-3184 (2003).

13. Romero, J., Lousa, A., Martinez, E., Esteve J.: Nanometric chromium/chromium carbide multilayers for tribological applications. Surface Coatings Technol. 163, 392-397 (2003).

14. Voevodin, A. A., Zabinski, J. S.: Superhard, functionally gradient, nanolayerd and nanocomposite diamond-like carbon coatings for wear protection. Diamond and Related Mater. 7, 463-467 (1998).

15. Gouldstone, A., Chollacoop, N., Dao, M., Li, J., Minor, A. M., Shen, Y.-L.: Indentation across size scales and disciplines: Recent developments in experimentation and modeling. Acta Mater. 55, 4015-4039 (2007).

16. Tan, X. H., Shen, Y.-L.: Modeling analysis of the indentation-derived yield properties of metallic multilayered composites. Composites Sci. Technol. 65, 1639-1646 (2005).

17. Jones, R. M.: Mechanics of Composite Materials. pp.37. Scripta Book Company, Washington, D.C (1975)

18. Shen, Y.-L., Finot, M., Needleman, A., Suresh, S.: Effective elastic response of two-phase composites. Acta Metall. Mater. 42, 77-97 (1994).

19. Wilkins, T., Shen, Y.-L.: Stress enhancement at inclusion particles in aluminum matrix composites: Computational modeling and implications to fatigue damage. Computational Mater. Sci. 22, 291-299 (2001).

20. Chawla, N., Chawla, K. K.: Metal Matrix Composites. pp.146.Springer, New York (2006)

21. Fischer-Cripps, A. C.: Nanoindentation. pp.20-21. Springer, New York (2002)

22. Bucaille, J. L., Stauss, S., Schwaller, P., Michler, J.: A new technique to determine the elastoplastic properties of thin metallic films using sharp indenters. Thin Solid Films. 447, 239-245 (2004).

23. Lide, D. R.: Handbook of Chemistry and Physics, 76th ed., CRC Press (1995).

24. Oliver, W. C., Pharr, G. M.: An improved technique for determining hardness and elastic-modulus using load and displacement sensing indentation experiments. J. Mater. Res. 7, 1564-1583 (1992).

25. Cheng, Y.-T., Cheng, C.-M.: Can stress-strain relationship be obtained from indentation curves using conical and pyramidal indenters? J. Mater. Res. 14, 3493-3496 (1999).

26. Gao, H., Chiu, C.-H., Lee, J.: Elastic contact versus indentation modeling of multi-layered materials. Int. J. Solids Structures. 29, 2471-2492 (1992).

\section{GUANLIN TANG}

Ms. Tang is a Ph.D candidate in the Department of Mechanical Engineering at University of New Mexico. Her research interests are in the solid mechanics and nano-material science areas, including computational mechanics, finite element modeling and mechanical behavior of nano-composites.

\section{YU-LIN SHEN}

Dr. Shen is currently a professor in the Department of Mechanical Engineering at University of New Mexico. His research interests include mechanical behavior of materials, multiscale computational modeling, and mechanics issues in thin films, composites and microelectronic devices/packages. He is a Fellow of ASME (American Society of Mechanical Engineers). 